\title{
A GRAPH AND ITS COMPLEMENT WITH SPECIFIED PROPERTIES III: GIRTH AND CIRCUMFERENCE
}

\author{
JIN AKIYAMA AND FRANK HARARY \\ Department of Mathematics \\ The University of Michigan \\ Ann Arbor, Michigan 48109 U.S.A.
}

(Received April 5, 1979)

ABSTRACT. In this series, we investigate the conditions under which both a graph $G$ and its complement $\bar{G}$ possess certain specified properties. We now characterize all the graphs $G$ such that both $G$ and $\bar{G}$ have the same girth. We also determine all $G$ such that both $G$ and $\bar{G}$ have circumference 3 or 4 .

KEY WORDS AND PHRASES. Graph, Complement, Girth, Circumference.

1980 MATHEMATICS SUBJECT CLASSIFICATION CODES. $05 C 99$.

${ }^{1}$ Visiting Scholar, 1978-79, from Nippon Ika University, Kawasaki, Japan.

${ }^{2}$ Vice-President, Calcutta Mathematical Society, 1978 and 1979. 
1. NOTATIONS AND BACKGROUND.

In the first paper [2] in this series, we found all graphs $G$ such that both $G$ and its complement $\bar{G}$ have (a) connectivity 1 , (b) line-connectivity 1 , (c) no cycles, (d) only even cycles, and other properties. Continuing this study, we determined in [3] the graphs $G$ for which $G$ and $\bar{G}$ are (a) block-graphs, (b) middle graphs, (c) bivariegated, and (d) n'th subdivision graphs. Now we concentrate on the two invariants concerning cycle lengths: girth and circumference. We will see that whenever $G$ and $\bar{G}$ have the same girth $g$, then $\mathrm{g}=3$ or 5 only. For the circumference $\mathrm{c}$, we study only the cases where both $G$ and $\bar{G}$ have $\mathrm{c}=3$ or 4 .

Following the notation and terminology of [4], the join $G_{1}+G_{2}$ of two graphs is the union of $G_{1}$ and $G_{2}$ with the complete bigraph having point sets $V_{1}$ and $V_{2}$. We will require a related ternary operation denoted $G_{1}+G_{2}+G_{3}$ on three disjoint graphs, defined as the union of the two joins $G_{1}+G_{2}$ and $\mathrm{G}_{2}+\mathrm{G}_{3}$. Thus, this resembles the composition of the path $\mathrm{P}_{3}$ not with just one other graph but with three graphs, one for each point; Figure 1 illustrates the "random" graph $\mathrm{K}_{4}-\mathrm{e}=\mathrm{K}_{1}+\mathrm{K}_{2}+\mathrm{K}_{1}$. Of course the quaternary operation $G_{1}+G_{2}+G_{3}+G_{4}$ is defined similarly.

Recall that the corona $G \circ H$ of two graphs $G$ with $p$ points $v_{i}$, and $H$ is obtained from $G$ and $p$ copies of $H$ by joining each point $v_{\hat{1}}$ of $G$ with all the points of the $i^{\prime}$ th copy of $H$. Again, for our result on girth we need a ternary operation written $G_{1}+G_{2} \circ G_{3}$ which is defined as the union of the join $G_{1}+G_{2}$ with the corona $G_{2} \circ G_{3}$. For example, Figure 2 illustrates the graph $A=K_{1}+K_{2} \circ K_{1}$.

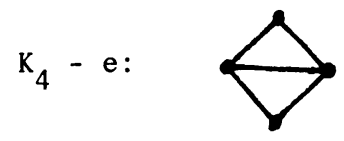

Figure 1. $\quad K_{4}-e=K_{1}+K_{2}+K_{1}$ 
A :

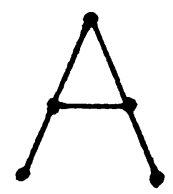

Figure 2. $\quad A=K_{1}+K_{2} \circ K_{1}$

\section{GIRTH}

The girth of a graph $G$, denoted by $g=g(G)$, is the length of a shortest cycle (if any) in G. Note that this invariant is undefined if $G$ has no cycles. For instance, the tetrahedron $K_{4}$, the 3-cube $Q_{3}$ and the Petersen graph $P$ illustrated in Figure 3 have girth 3,4 and 5 , respectively.
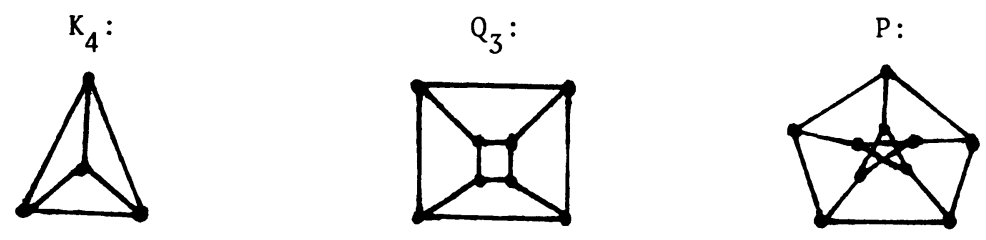

Figure 3. Graphs with small girth

Let $\bar{g}$ denote $g(\bar{G})$. In order to find all graphs $G$ with $g=\bar{g}$, we first develop two lemmas dealing with $g \geq 4$ and with $g=3$.

LEMMA 1. There are no graphs $G$ other than $C_{5}$ such that both $G$ and $\bar{G}$ have girth at least 4 .

PROOF. If the number of points of $G$ is at least 6 , then $G$ or $\bar{G}$ contains $C_{3}$ since the ramsey number $r\left(C_{3}\right)=6$; see $[4, p .16]$. On the other hand, the only graphs $G$ with at most 5 points and of girth at least 4 are $\mathrm{C}_{4}, \mathrm{C}_{4} \boldsymbol{U} \mathrm{K}_{1}, \mathrm{C}_{4} \cdot \mathrm{K}_{2}$ and $\mathrm{C}_{5}$. However, none of their complements except $\mathrm{C}_{5}$ has girth at least 4 . 
LEMMA 2. If both $G$ and $\bar{G}$ contain a triangle, then there are two triangles, one in $G$ and the other in $\bar{G}$, which have exactly one common point.

PROOF. Take any pair of triangles $T_{1}$ from $G, T_{2}$ from $\bar{G}$. Obviously, $\mathrm{T}_{1}$ and $\mathrm{T}_{2}$ can have at most one common point. Since the lemma is trivial if $\mathrm{T}_{1}$ and $\mathrm{T}_{2}$ have a common point, we may assume that $\mathrm{T}_{1}$ and $\mathrm{T}_{2}$ have no common points. Color the lines of $\mathrm{T}_{1}$ and $\mathrm{T}_{2}$ with green and red, respectively. Consider the complete bigraph $\mathrm{K}_{3,3}$ whose point sets are $\mathrm{V}\left(\mathrm{T}_{1}\right)$ and $\mathrm{V}\left(\mathrm{T}_{2}\right)$, and color the lines of $\mathrm{K}_{3,3}$ with either green or red arbitrarily. Since there are in $\mathrm{K}_{3,3}$ at least 5 lines of the same color, say green, there is a point of $\mathrm{V}\left(\mathrm{T}_{2}\right)$ with which two green lines of $\mathrm{K}_{3,3}$ are incident. Thus, these two lines and a line of $\mathrm{T}_{1}$ determine a green triangle in $\mathrm{G}$ which has a common point $\mathrm{V}$ with the red triangle $\mathrm{T}_{2}$ in $\overline{\mathrm{G}} \cdot \mathrm{C}$

We can restate Lemma 2 in terms of acquaintances at a party. At any party with at least five people where there are three mutual acquaintances and three mutual strangers, there must be a person who is acquainted with a pair of mutual acquaintances and who is acquainted with neither of two mutual strangers.

A subject related to Lemma 2 is discussed in [5], which specifies all the cases such that there are exactly two monochromatic triangles in the 2 -colorings of $\mathrm{K}_{6}$.

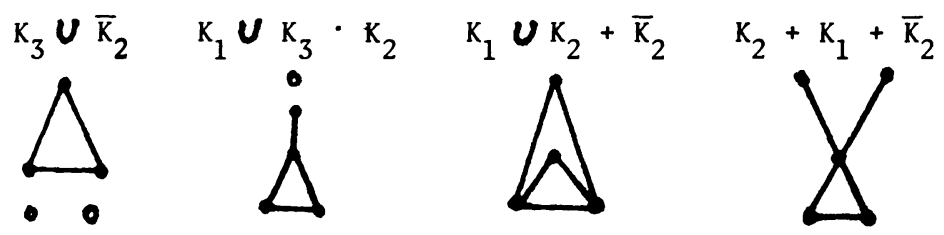



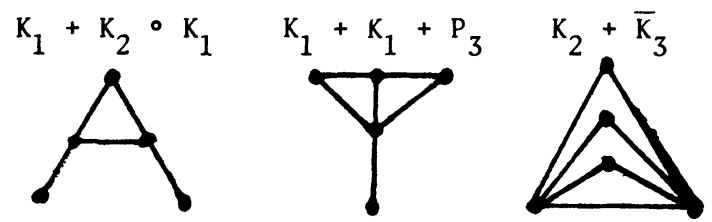

Figure 4. The seven graphs of the iff-induced family for the set of all graphs $\mathrm{G}$ with $g=\bar{g}=3$.

Consider two family of graphs $\stackrel{N}{=}$ and $\stackrel{H}{=} \cdot$ In [1], the letter $\stackrel{N}{=}$ was chosen to stand for "necessary subgraphs". However, for the purpose of specifying a11 graphs $G$ with $g=\bar{g}=3$, we require the family $\stackrel{N}{=}$ to be both necessary and sufficient in the following sense. We say that $N$ is an iff-induced family of graphs for $\stackrel{H}{=}$ if:

a) every graph in $\underset{x}{H}$ contains some graph in $\stackrel{N}{=}$ as an induced subgraph; and

b) every graph $\mathrm{G}$ containing some graph in $\stackrel{N}{=}$ as an induced subgraph must be in $\stackrel{H}{=}$.

We illustrate with Beineke's characterization of line graph in terms of the set $\stackrel{N}{=}$ of nine forbidden induced subgraphs shown in $[4, p .75]$. Let $\stackrel{H}{=}$ be the family of all graphs which are not line graphs. Then this set $\stackrel{N}{N}$ is an iff-induced family for $\stackrel{H}{=}$.

THEOREM 1. Let $\stackrel{H}{=}$ be a family of graphs with $g=\bar{g}=3$. Then the set of seven graphs $\mathrm{K}_{3} U \overline{\mathrm{K}}_{2}, \mathrm{~K}_{1} U \mathrm{~K}_{3} \cdot \mathrm{K}_{1}, \mathrm{~K}_{1} U \mathrm{~K}_{2}+\overline{\mathrm{K}}_{2}, \mathrm{~K}_{2}+\mathrm{K}_{1}+\dot{\overline{\mathrm{K}}}_{2}, \mathrm{~K}_{1}+\mathrm{K}_{2} \cdot \mathrm{K}_{1}$, $K_{1}+K_{1}+P_{3}$ and $K_{2}+\bar{K}_{3}$ is an iff-induced family for $\stackrel{H}{=}$.

PROOF. When $\mathrm{g}=\overline{\mathrm{g}}=3$, by definition both $\mathrm{G}$ and $\overline{\mathrm{G}}$ contain a triangle. By Lemma 2, there is a set $U$ of five points of $G$ such that both the induced subgraphs $\langle\mathrm{U}\rangle$ in $\mathrm{G}$ and in $\overline{\mathrm{G}}$ contain triangles. A graph $\mathrm{F}$ of order 5 such that both $F$ and $\bar{F}$ contain a triangle is one of the 7 graphs in Figure 4 . Thus, the sufficiency is proved. Since each of the seven graphs and their complements contain a triangle, the necessity also holds. [ 


\section{CIRCUMFERENCE}

It is now natural to consider the circumference $c=c(G)$, the length of a longest cycle in $G$, in place of the girth. However, as it is known that almost all graphs are hamiltonian, see Wright [7], this question is hopeless in general since there will be too many graphs $G$ such that both $G$ and $\bar{G}$ have circumference $p$, the number of points of $G$. Hence, we now ask this question only for $\mathrm{c}=3$ and 4 .
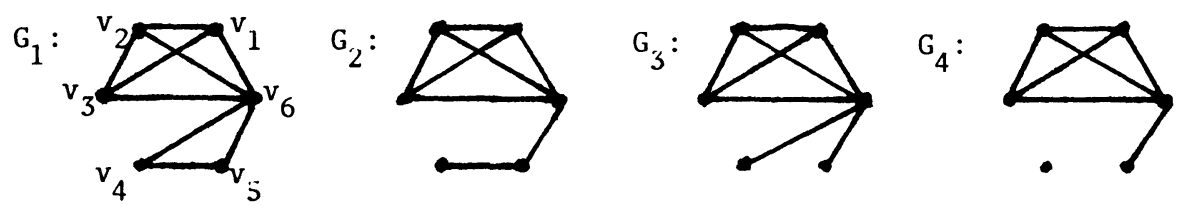

$\mathrm{G}_{5}$ :
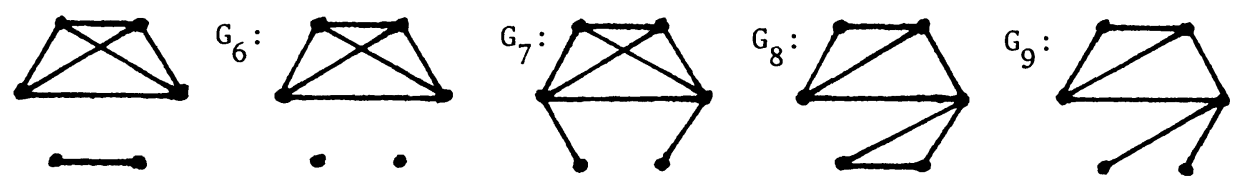

Figure 5. Graphs with $c=\bar{c}=4$

THEOREM 2. The graph $A=K_{1}+K_{2} \circ K_{1}$ is the only graph with $c=\bar{c}=3$. A11 the eighteen graphs with $c=\bar{c}=4$ are $G_{1}=K_{4} \cdot K_{3}, G_{2}=K_{1}+K_{1}+K_{1}+K_{3}$, $\mathrm{G}_{3}=\bar{K}_{2}+\mathrm{K}_{1}+\mathrm{K}_{3}, \mathrm{G}_{4}=\mathrm{K}_{1} \boldsymbol{U} \mathrm{K}_{1}+\mathrm{K}_{1}+\mathrm{K}_{3}, \mathrm{G}_{5}=\mathrm{K}_{2} \boldsymbol{U} \mathrm{K}_{4}, \mathrm{G}_{6}=\mathrm{K}_{4} \boldsymbol{U} \bar{K}_{2}$, $\mathrm{G}_{7}=\mathrm{K}_{2}+\mathrm{K}_{2} \circ \mathrm{K}_{1}, \mathrm{G}_{8}=\mathrm{K}_{2}+\mathrm{K}_{1}+\mathrm{K}_{2}+\mathrm{K}_{1}$, and $\mathrm{G}_{9}=\bar{K}_{2}+\mathrm{K}_{1}+\mathrm{K}_{2}+\mathrm{K}_{1}$ and their complements.

PROOF. We first settle the condition $c=\bar{c}=3$. This precludes graphs $G$ of order $p \geq 6$ since the ramsey number $r\left(C_{4}\right)=6$ as mentioned in [6]. Hence, when $\mathrm{p} \geq 6, \mathrm{G}$ or $\overline{\mathrm{G}}$ contains $\mathrm{C}_{4}$ and so has circumference at least 4 . Thus, if $c=\bar{c}=3$, then $p \leq 5$. But as $K_{4}$ does not have two line-disjoint triangles, we also have $p \geq 5$. Thus, it is sufficient to consider graphs with exactly 
five points. It is easily verified that the only graph with $c=\bar{c}=3$ is $A=K_{1}+K_{2} \circ K_{1}$ among all graphs of order 5 .

We now find all the graphs $G$ with $c=\bar{c}=4$. Since $K_{5}$ does not contain two line-disjoint 4-cycles, the number of points $p(G) \geq 6$. We see by exhaustion that there are exactly 18 graphs $G$ of order 6 such that neither $G$ nor $\bar{G}$ contains $C_{5}$ or $C_{6}$, namely the nine graphs $G_{i}$ in Figure 5 and their complements $\bar{G}_{i}$. It is easily verified that all of them satisfy $c=\bar{c}=4$ Assume that there exists a graph $H$ of order 7 such that $c=\bar{c}=4$. Then the graph $G$ obtained by removing a point $V$ of $H$ must be one of the 18 graphs $G_{i}$ or $\bar{G}_{i}, i=1,2, \ldots, 9$. However, we now show that there are no graphs $H$ of order 7 such that neither $H$ nor $\bar{H}$ contains a cycle of length at least 5 and $H-v$ is one of the $G_{i}$ or $\bar{G}_{i}$. We label the points $v_{1}, v_{2}, v_{3}, v_{4}, v_{5}$ and $v_{6}$ of each $G_{i}$ or $\bar{G}_{i}$ just as in $\bar{G}_{1}$ in Figure 5 , and denote by $v$ the point of $H$ not belonging to $G_{i}, i=1,2, \ldots, 9$. It is convenient to divide the proof into two cases.

CASE 1. Either $\mathrm{H}-\mathrm{v}$ or $\overline{\mathrm{H}-\mathrm{v}}$ is one of the $\mathrm{G}_{i}, \mathbf{i}=1,2, \ldots, 7$. Without loss of generality, we may assume that $H-v$ is one of the $G_{i}, i=1,2, \ldots, 7$. We see that there are paths of length 3 or 4 joining $v_{j}$ and $v_{k}$ in both $G_{i}$ and $\bar{G}_{i}$ for any distinct points $v_{j}$ and $v_{k}, 1 \leq j, K \leq 3$. The point $v$ must be adjacent to at least two points $v_{j}, j=1,2,3$, in either $H$ or $\bar{H}$. Thus either $\mathrm{H}$ or $\overline{\mathrm{H}}$ contains $\mathrm{C}_{5}$ or $\mathrm{C}_{6}$, which is a contradiction.

CASE 2. Either $\mathrm{H}-\mathrm{v}$ or $\overline{\mathrm{H}-\mathrm{v}}$ is $\mathrm{G}_{8}$ or $\mathrm{G}_{9}$. There are two possibilities. If $v$ is adjacent to $v_{2}$ in $H$, then $v$ is forced to be nonadjacent to $v_{3}$ in $H$, since in $G_{i}$ there is a path of length 3 joining $v_{2}$ and $v_{3}$. There is also a path of length 3 in $G_{i}$ joining $v_{2}$ and $v_{6}$, and one in $\bar{G}_{i}$ joining $v_{3}$ and $v_{6}$. Hence either $H$ or $\bar{H}$ contains $\mathrm{C}_{5}$, which is a contradiction. 
On the other hand, if $v$ is not adjacent to $v_{2}$ in $H$, then $v$ is forced to be adjacent to $v_{3}$ in $G_{i}$ since in $\bar{G}_{i}$ there is a path of length 4 joining $v_{2}$ and $v_{3}$. As there is a path in $G_{i}$ of length 3 joining $v_{3}$ and $v_{6}, v$ is forced to be nonadjacent to $v$ in $H$. Independent of the adjacency of $v$ and $v_{4}$ in $H$, either $H$ or $\bar{H}$ contains $C_{5}$, a contradiction. Since there are no graphs of order 7 with $c=\bar{c}=4$, no graph of greater order can satisfy this condition. []

\section{REFERENCES}

1. Akiyama, J., G. Exoo and F. Harary Covering and packing in graphs IIl:

Cyclic and acyclic invariants. Math. Slovaca (to appear).

2. Akiyama, J. and F. Harary A graph and its complement with specified properties I. Int'1. J. Math. and Math. Physics (to appear).

3. Akiyama, J. and F. Harary A graph and its complement with specified properties II. Nanta Math. (to appear).

4. Harary, F., Graph Theory. Addison-Wesley, Reading (1969).

5. Harary, F. The two-tiiangle case of the acquaintance graph. Math. Mag. 45 (1972) $130-135$.

6. Harary, F. A survey of generalized ramsey theory. Graphs and Combinatorics (R. Bari and F. Harary, eds.) Springer Lecture Notes 406 (1973) 10-17.

7. Wright, E. M. The proportion of unlabelled graphs which are hamiltonian. Bul1. London Math. Soc. 8 (1976) $241-244$. 


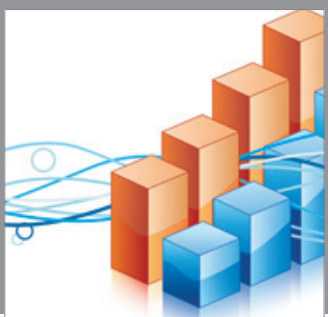

Advances in

Operations Research

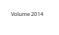

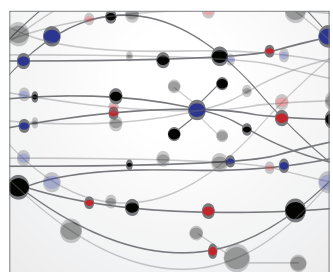

\section{The Scientific} World Journal
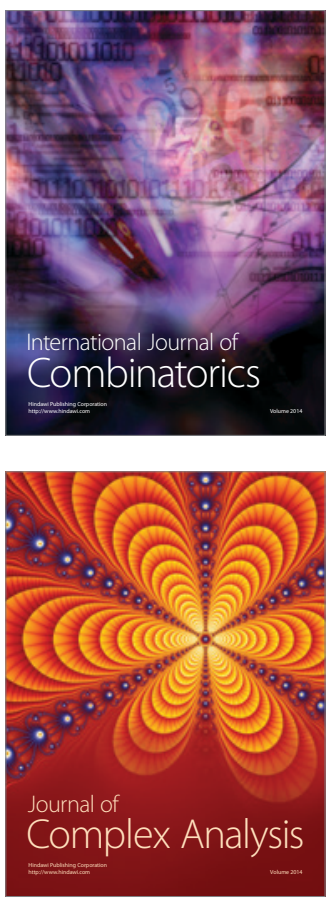

International Journal of

Mathematics and

Mathematical

Sciences
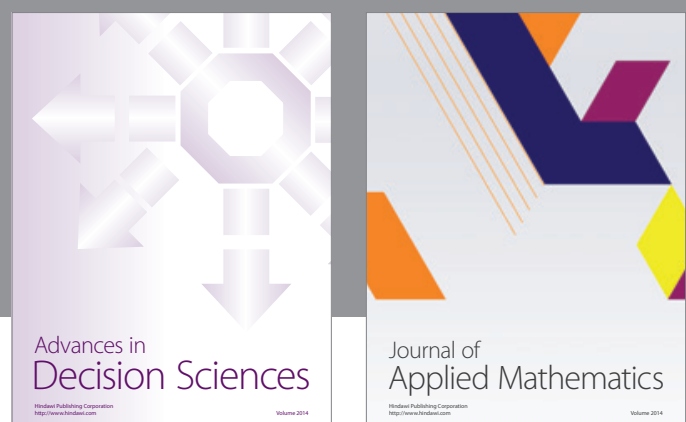

Journal of

Applied Mathematics
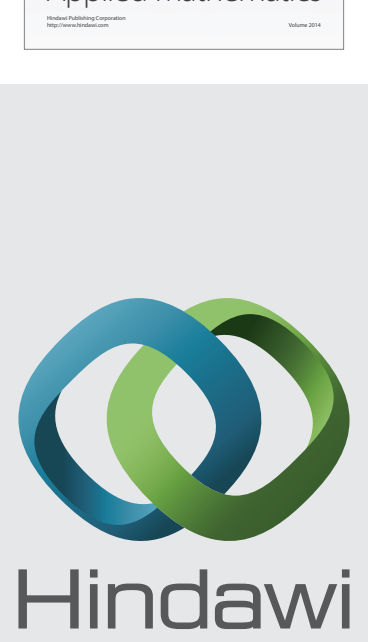

Submit your manuscripts at http://www.hindawi.com
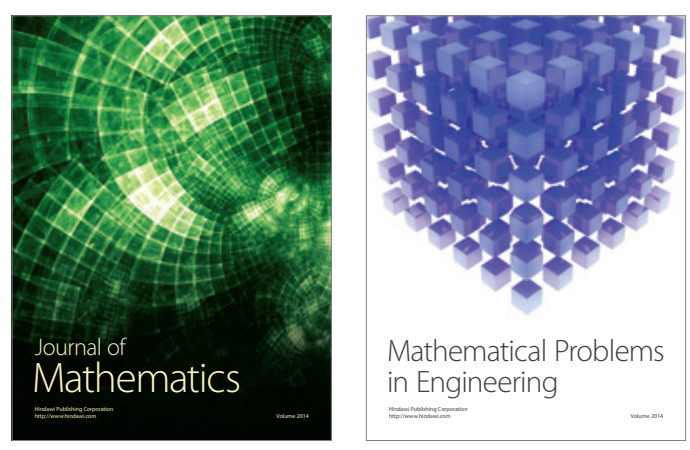

Mathematical Problems in Engineering
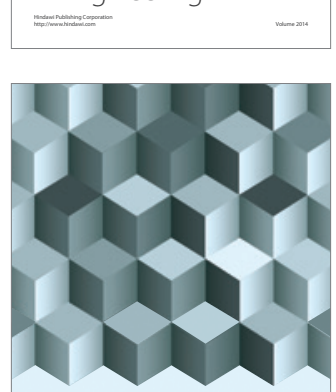

Journal of

Function Spaces
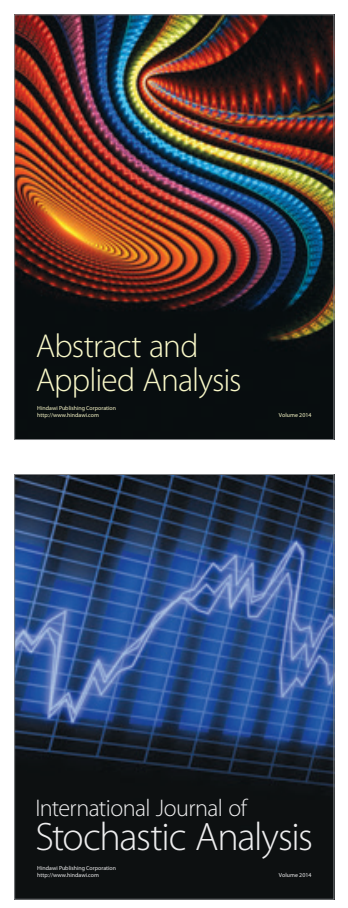

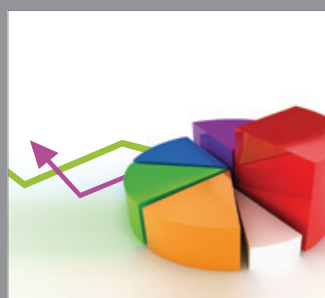

ournal of

Probability and Statistics

Promensencen
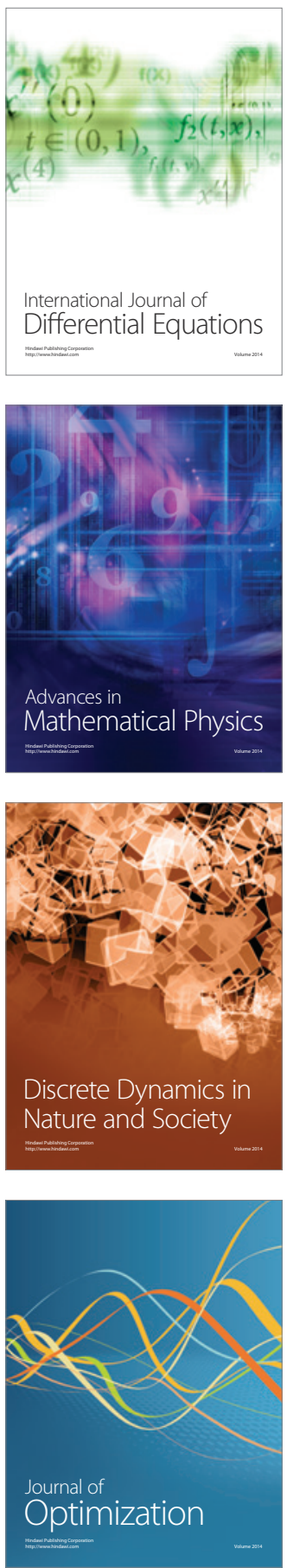